\title{
Proton Improves BED Compared With Photon Beam- based Partial Stereotactic Ablative Boost Radiotherapy for Large Non-small Cell Lung Cancer
}

\section{Yun Bai}

Peking University First Hospital

\section{Zhilei Zhao}

Yi Zhou International Proton Therapy Medical Center

Xianshu Gao ( $\nabla$ doctorgaoxs@126.com )

Peking University First Hospital https://orcid.org/0000-0002-8348-456X

Shangbin Qin

Peking University First Hospital

Siwei Liu

Peking University First Hospital

Bo Zhao

Peking University First Hospital

Mingwei Ma

Peking University First Hospital

\section{Xueying Ren}

Peking University First Hospital

\section{Xiaoying Li}

Peking University First Hospital

Xin Qi

Peking University First Hospital

Jiayan Chen

Peking University First Hospital

Mu Xie

Peking University First Hospital

\section{Xuanfeng Ding}

Beaumont Health System: Beaumont Health

\section{Research}

Keywords: Proton, Photon, Stereotactic Ablative Radiotherapy, NSCLC, Large tumor 
Posted Date: October 16th, 2020

DOl: https://doi.org/10.21203/rs.3.rs-92220/v1

License: (c) (i) This work is licensed under a Creative Commons Attribution 4.0 International License. Read Full License 


\section{Abstract}

Purpose: Increased volume ratio of Biologic Effective Dose (BED) was associated with higher local control rate in a setting of large (greater than $5 \mathrm{~cm}$ ) non-small cell lung cancer (NSCLC) via photon partial stereotactic ablative boost radiotherapy (P-SABR). Taking advantage of unique physics characteristic of the Bragg peak, we exploit the potential dosimetric and clinical benefit of using proton P-SABR.

Methods: Thirty patients' CT structure set with large NSCLC previously treated with photon P-SABR were re-planned with three different treatment modalities: intensity modulated proton therapy (Proton-IMPT), proton volumetric modulated arc therapy (Proton-Arc) and photon volumetric modulated arc therapy (Photon-VMAT). Proton-Arc was mimicked with 8 to 14 beams (10 to 20 degree apart). Dosimetric metrics were compared among all of three treatment modalities in which tumor and organ at risks (OARs) doses were compared in volumes receiving $>50 \%$ and $\leq 50 \%$ of the prescription dose, representing high- and low-dose regions, respectively. Normal Tissue Complication Probability (NTCP) of lungs and esophagus were compared as well.

Results: In the high-dose region, mean doses of PTVs were 72.64 GyE, 80.42 GyE and 82.22 GyE and $\mathrm{B}_{90}$ (the ratio of BED>90GyE to the in-field tumor) were $71.17 \%, 86.59 \%$ and $87.32 \%$ for Photon-VMAT, ProtonIMPT and Proton-Arc plans, respectively (photon versus proton, $p<0.05$ ). In addition, proton plans could keep a similar OARs dose sparing in high-dose region. In stage N2 to N3 group, $\mathrm{V}_{50}$ of esophageal were comparable for the three treatment modalities $(p>0.05)$. In the low-dose region, doses of OARs were reduced dramatically in proton plans compared with Photon-VMAT plans $(p<0.05)$. The potential clinical benefit of utilizing Proton-IMPT will reduce the mean probability of symptomatic radiation pneumonitis by $61.63 \%$ and the mean probability of grade $\geq 2$ radiation-induced esophagitis by $50.00 \%$ in comparison with Photon-VMAT group $(\mathrm{p}<0.05)$.

Conclusion: Higher BED inside the target could be achieved by using proton beam therapy with similar OARs dose sparing in high-dose region and significantly better OARs low dose sparing compared with photon techniques. Proton techniques has a potential role in the management of large NSCLC to increase the local control rate while reducing the side effect.

\section{Background}

It is generally challenging to achieve effective local control in large lung cancer (larger than $5 \mathrm{~cm})^{[1]}$. Stereotactic ablation radiotherapy (SABR), which could deliver very high Biologic Effective Dose (BED) to the tumor target $[2,3]$, has emerged as a promising therapeutic alternative to achieve high local control in early stage of cancer ${ }^{[4,5]}$. However, patients with large NSCLC are unable to tolerate SABR ${ }^{[6-9]}$.

In one approach to overcome this, patients with large tumor are treated on the combination of SABR with conventionally fractionated radiotherapy (CFRT) ${ }^{[10-13]}$. According to our previous research ${ }^{[13]}$, partial stereotactic ablative boost radiotherapy (P-SABR) could optimize BED with equivalent OARs doses, thus 
increase local control of large NSCLC significantly with tolerable toxicity. In addition, patients with higher $B_{90}$ (the ration of $B E D>90 G y E$ to the in-field tumor) could achieve higher local control rate. How to improve $B_{90}$ while decrease organ at risk (OAR) dose is the focus of research.

There is currently an increasing interest in proton radiotherapy due to the dosage advantage of Bragg peak $^{[14]}$. Proton CFRT can increase the target dose regardless of the dose ceiling while reduce the OAR dose significantly compared with photon therapy in unresectable stage III NSCLC ${ }^{[15]}$. Therefore, this study intends to explore whether proton P-SABR can deliver higher $\mathrm{B}_{90}$ compared with photon plans in large NSCLC tumors.

Study on early stage of NSCLC further showed that only low-dose region is decreased in proton plan compared with photon plan while high-dose region is higher in proton SABR plan due to uncertainty of proton beams ${ }^{[16]}$. However, Proton Arc Therapy (Proton-Arc) could achieve smaller high-dose region than Intensity Modulated Proton Therapy (Proton-IMPT) in early stage of NSCLC ${ }^{[17]}$. Therefore, our research focus on Proton-Arc and Proton-IMPT plans as well.

\section{Methods}

Patients

Thirty patients with large NSCLC who previously received P-SABR with photon beams were included in the present study. Two patients (6.7\%) had stage IIB(T3NOM0), 17 patients $(56.7 \%)$ had stage III and 11 patients (36.7\%) had stage IV according to AJCC Cancer Staging Manual, Seventh Edition. Patients with N0, N1, N2, N3 stage were 8 (26.7\%), 4 (13.3\%), 8 (26.7\%), 9 (30.0\%), respectively. Median volume of the tumor treated by P-SABR was $138.07 \mathrm{~cm}^{3}\left(36.2\right.$ to $\left.95.50 \mathrm{~cm}^{3}\right)$ with a median maximal diameter of $8.08 \mathrm{~cm}$ $(4.62$ to $14.64 \mathrm{~cm}$ ). (Table 1) In our study organ motion was not an issue because of the bulky tumor volume. However, to control for motion effects in proton delivery, we intended to use tumor gating.

\section{Table 1 Patients and treatment characteristics}




\begin{tabular}{|c|c|c|c|}
\hline Characteristic & Total N (\%) & Characteristic & Total N (\%) \\
\hline Patient characteristics $(n=3$ & patients) & Staging & \\
\hline Gender & & IIB & $2(6.7 \%)$ \\
\hline Male & $28(93.3 \%)$ & IIIB & $17(56.7 \%)$ \\
\hline Female & $2(6.7 \%)$ & IV & $11(36.7 \%)$ \\
\hline Target Location & & Tumor volume $\left(\mathrm{cm}^{3}\right)$, & 138.1 \\
\hline Right Upper & $9(30 \%)$ & median (range) & $(35.2-495.5)$ \\
\hline Right Lower & $4(13.3 \%)$ & Tumor size $(\mathrm{cm})$, & 8.1 \\
\hline Left Upper & $10(33.3 \%)$ & median (range) & $(4.6-14.6)$ \\
\hline Left Lower & $7(23.3 \%)$ & SABR plans & \\
\hline Histology & & Single dose (GyE/ fraction), & 6.0 \\
\hline Squamous cell carcinoma & $16(53.3 \%)$ & median (range) & $(4.0-8.0)$ \\
\hline Adenocarcinoma & $6(20.0 \%)$ & Fractions, & 4 \\
\hline Others & $4(13.3 \%)$ & median (range) & $(3-6)$ \\
\hline Unknown & $4(13.3 \%)$ & Total dose (GyE), & 24.0 \\
\hline T stage & & median (range) & $(15.0-48.0)$ \\
\hline T2 & $8(26.7 \%)$ & CFRT plans & \\
\hline T3 & $11(36.7 \%)$ & Single dose (GyE/ fraction), & 2.0 \\
\hline T4 & $11(36.7 \%)$ & median (range) & $(1.8-3.0)$ \\
\hline N stage & & Fractions, median (range) & $26(11-33)$ \\
\hline NO & $8(26.7 \%)$ & Total dose (GyE), & 52.0 \\
\hline N1 & $4(13.3 \%)$ & median (range) & $(33.0-66.0)$ \\
\hline N2 & $8(26.7 \%)$ & Total doses & \\
\hline N3 & $9(30.0 \%)$ & GTVb margin (GyE), & 76.0 \\
\hline M stage & & median (range) & $(69.0-87.0)$ \\
\hline MO & $19(63.3 \%)$ & CTV margin (GyE), & 64.0 \\
\hline M1 & $11(16.7 \%)$ & median (range) & $(51.0-75.0)$ \\
\hline
\end{tabular}




\section{Contouring}

Targets and OARs delineation in photon plans were described previously[13]. The gross tumor boost volume (GTVb) of photon was the max volume receiving SABR while ensure the fatal OARs dose falloff to $3 \mathrm{GyE} / \mathrm{f}$. It should be noted that, for each patient, individualized GTVb-proton was designed to reach the maximal deliverable dose while fatal OARs dose falloff to 3 GyE/f. GTV, ITV, CTV and PTV were contoured identically for protons and photons, while the PTV of proton plan was just for comparison. (Figure 1)

Dose

The doses of photon P-SABR were shown as before ${ }^{[13]}$ and the doses of proton P-SABR were similar. In short, P-SABR plan consisted of a SABR plan to GTVb followed by a CFRT plan to CTV. The dose of photon-GTVb and proton-GTVb margin were both 15 to 48GyE in 3 to 6 fractions (Table 1). It should be mentioned that, by definition, center dose of GTVb should be as high as possible regardless of homogeneity, which means significant high-dose was introduced inside the target volume. And the dose of CTV was 33 to $66 \mathrm{GyE}$ in 11 to 33 fractions which was equal for all three techniques. The total mean doses of GTVb and CTV margin were 76GyE and 64GyE, respectively.

\section{Proton Plan}

Pencil beam proton planning was performed using RayStation (V6.0, Raysearch Laboratories Inc) with automatic spot spacing and spot placing. Patients were re-planned with both Proton-IMPT and Proton-Arc plans. Proton-IMPT plans used two to three coplanar beams, while Proton-Arc were mimicked with 8 to 14 beams placed at approximately $10^{\circ}$ to $20^{\circ}$ increments. The beams were coplanar and selected to maximize access to the tumor while minimizing exposure to normal tissues. Monte Carlo dose calculation algorithm was used for proton dose calculation with a 70-230 MeV energy range. A maximum of $0.3 \mathrm{~cm}$ uniform setup error and a range uncertainty of $3.5 \%$ were used in the robustness setting for optimization. The similar objectives and constraints for organs OARs were used for both photon and proton plans. Both proton plans were normalized for $99 \%$ of the CTVs covered by $100 \%$ of the prescription dose. Normal tissue dose constraints (lung, heart, esophagus and spinal cord) in composite plans were based on QUANTEC guideline ${ }^{[18]}$.

\section{Photon Plan}

The treatment planning was performed by Eclipse (V13.5, Varian Medical Systems) calculating by Analytical Anisotropic Algorithm (AAA) dose calculation algorithms. All patients were treated with $6 \mathrm{MeV}$ photons. Each patient underwent repeat planning with photon volumetric modulated arc therapy (PhotonVMAT) performed by a $180^{\circ}$ to $360^{\circ}$ arc. To match the proton plan target coverage, the planning goals for both photon treatments were as follows: $100 \%$ of the prescription dose covering $99 \%$ of the CTVs

Plan evaluation 
All proton and photon treatment plans were compared in terms of dose conformity, dose homogeneity, isodose coverage, BED and OAR sparing. Proton doses were corrected with relative biologic effectiveness (RBE) value of $1.1^{[19]}$. $D_{x}$ means the maximize dose received by $X \%$ volume. For example: $D_{02}$ means the maximize dose received by $2 \%$ volume. $V_{X}$ means the percentage of the structure receiving doses greater than $X$ does. For example: $V_{50}$ means the volume of the structure receiving doses greater than $50 \mathrm{GyE}$. $B E D$ was calculated using the linear- quadratic equation. $B_{X}$ means the volume of the structure receiving doses greater than $\mathrm{X}$ does. For example: $\mathrm{B}_{90}$ means the volume of the structure receiving doses greater than $90 \mathrm{GyE}$.

$B E D=N \times d \times(1+d /(\alpha / \beta))(N$ : fractionation number, d: daily dose, $a / \beta$ : 10 for NSCLC)

$\mathrm{V}_{\mathrm{X} \%}$ means the volume of all organs (tumor and OARs) that receive $\geq \mathrm{X} \%$ of the prescription dose. To compare the effect of proton and photon beams, we analyzed dose distributions in 2 separated parts, "high-dose" and "low-dose" regions. High-dose region is composed of all organs (tumor and/or OAR) that receive $>50 \%$ of the prescription dose $\left(\mathrm{V}_{50 \%}\right.$ in short). Low-dose region is composed of the organs that are outside the $50 \%$ prescription dose.

The degree of conformality for proton and photon plans was evaluated by calculating the RTOG conformity index $(\mathrm{Cl})$, defined as

$\mathrm{Cl}=\mathrm{V}_{95 \%} / \mathrm{V}_{\mathrm{PTV}}$

A conformity index equal to 1 corresponds to ideal conformation. A conformity index greater than 1 indicates that the irradiated volume is greater than the target volume and includes healthy tissues. If the conformity index is less than 1 , the target volume is only partially irradiated.

The homogeneity index $(\mathrm{HI})$ was evaluated by calculating the $\mathrm{D}_{98}$ to $\mathrm{D}_{2}$ ratio in this study to evaluate the homogeneity of the plans. The higher of the $\mathrm{HI}$, the plan is more homogeneous.

The benefits of proton plans in the clinical setting were estimated by Lyman-Kutcher-Burman (LKB) normal tissue complication probability (NTCP) model ${ }^{[20]}$.

$$
\mathrm{NTCP}=\frac{1}{\sqrt{2 \pi}} \int_{-\infty}^{t} e^{-\frac{x^{2}}{2}} d x, \quad \text { where } t=\frac{E U D-T D_{50}}{m T D_{50}}, \quad E U D=\left(\sum_{i} v_{i} D_{i}^{\frac{1}{n}}\right)^{n}
$$

in which EUD is the generalized equivalent uniform dose; $T D_{50}$ represents the dose tolerance of the whole organ and is associated with $50 \%$ complication risk; $m$ is a measure of the slope of the sigmoid curve represented by the integral of the normal distribution; $v_{i}$ is the relative volume that received a dose of $D_{i}$; and $n$ indicates the volume effect. When we used this model to predict the probability of symptomatic radiation pneumonitis within 6 months of radiation. ' $m$ ', 'TD50' and ' $n$ ' were set as 0.41 , 29.9Gy (relative biological effectiveness) and 1 , respectively ${ }^{[21]}$. When we used this model to predict the probability of 
grade $\geq 2$ radiation-induced esophagitis within 6 months from the first treatment. ' $m$ ', 'TD 50' and ' $n$ ' were set as $0.65,32.84 \mathrm{~Gy}$ (relative biological effectiveness) and 1.04, respectively ${ }^{[22]}$.

Statistical analysis

We used statistical software SPSS 25.0 for analyses. The plans were compared with regard to targets and OARs doses. One-way ANOVA was used for all statistical comparisons, with a $p$ values of $<0.05$ considered significant; $p$ values of $<0.001$ were truncated and noted as $p<0.001$.

\section{Results}

High-dose region analysis (PTV, $\mathrm{Cl}, \mathrm{HI}$, Esophagus)

\section{Target dose comparison}

The mean doses of photon-GTVb was 27.43GyE for Photon-VMAT, while the mean doses of proton-GTVb were able to escalate to 50.41GyE and 51.05GyE for Proton-IMPT and Proton-Arc, respectively, in 4 fractions ( 3 to 6 fractions) (photon vs. proton $(p<0.001)$ ). The mean doses of CTV were $55.27 \mathrm{GyE}, 53.96$ GyE and 55.51 GyE, respectively for Photon-VMAT, Proton-IMPT and Proton-Arc, which were equal for all three techniques $(p>0.1)$. The total mean doses of $\mathrm{GTVb}$ were $82.70 \mathrm{GyE}, 104.38 \mathrm{GyE}$ and $106.56 \mathrm{GyE}$, and the total mean BED of GTVb were 114.16 GyE, 181.44 GyE and 185.73 GyE, respectively for PhotonVMAT, Proton-IMPT and Proton-Arc (photon vs. proton $(p<0.001)$ ).

For dose of PTV, $D_{\text {mean }}, D_{02}, D_{\text {max }}, V_{75}, V_{80}, V_{85}, V_{90}, B_{90}, B_{100}, B_{110}$ and $B_{120}$ were higher for proton plans significantly (Figure 2 ), whereas $\mathrm{D}_{\min }$ of PTV were higher for Photon-VMAT plans $(\mathrm{p}<0.05)$. (Table 2$)$ It's worthy to mention that Proton-IMPT could increase $B_{90}, B_{100}, B_{110}$ and $B_{120}$ by $15.41 \%, 31.81 \%, 42.62 \%$, $46.57 \%$, compared to Photon-VMAT, respectively $(p<0.05)$.

Table 2 Comparison of doses to the target comparison between photon and proton plans

\begin{tabular}{|c|c|c|c|}
\hline & Photon-VMAT & Proton-IMPT & Proton-Arc \\
\hline$D_{\min }(G y E)$ & $45.74 \pm 15.09$ & $27.63 \pm 16.47^{\star \star \star}$ & $35.26 \pm 16.30^{*}$ \\
\hline $\mathrm{D}_{98}(\mathrm{GyE})$ & $60.10 \pm 9.97$ & $51.77 \pm 15.49^{*}$ & $52.66 \pm 14.82$ \\
\hline$D_{\text {mean }}(G y E)$ & $72.64 \pm 6.49$ & $80.42 \pm 11.04^{\star \star}$ & $82.22 \pm 9.80^{\star \star \star}$ \\
\hline$D_{02}(G y E)$ & $84.62 \pm 6.38$ & $112.38 \pm 17.13^{\star \star \star}$ & $114.52 \pm 11.81^{\star \star \star}$ \\
\hline$D_{\max }(G y E)$ & $87.29 \pm 6.56$ & $122.85 \pm 21.14^{\star \star \star}$ & $122.05 \pm 14.62^{\star \star \star}$ \\
\hline
\end{tabular}


Abbreviations: $\mathrm{D}_{\mathrm{X}}$ means the maximize dose received by $\mathrm{X} \%$ volume.

* means $p$ values of $<0.05$, ** means $p$ values of $<0.01$ and $* \star *$ means $p$ values of $<0.001$ compared with Photon-VMAT; There was no statistically significant difference between Proton-IMPT and Proton-Arc.

\section{Dose conformality and homogeneity}

Figure $3 \mathrm{a}, \mathrm{b}$ and $\mathrm{c}$ showed the Photon-VMAT, Proton-IMPT and Proton-Arc calculated dose distributions of one patient with large NSCLC, respectively. The DVH curves of each technique were shown in Figure 4. These results showed that proton plan could increase the target dose dramatically and reduce the OAR dose at the same time, while the dose distribution was less homogeneous than photon plans $(p<0.001)$, which was in consistence with HI. (Table 3 ) In addition, Proton-Arc plan was albeit more-conformal than Photon-VMAT and Proton-IMPT ( $p>0.05$ ). In high-dose regions, in spite of the $100 \%$ prescription isodose line $\left(V_{100 \%}\right)$ in proton plans encompasses generally larger volume compared with Photon-VMAT $(p>0.05)$, Proton-Arc plans could provide albeit lower $\mathrm{V}_{100 \%}$ than Proton-IMPT $(\mathrm{p}>0.05)$. Proton plans could reduce $\mathrm{V}_{50 \%}$ more significantly than Photon-VMAT plans $(\mathrm{p}<0.001)$. However, there was no significant difference between Proton-IMPT and Proton-Arc plan $(p>0.05)$.

Table 3 Comparison of target dosimetric metrics between photon and proton plans

\begin{tabular}{|llll|}
\hline Parameter & Photon-VMAT & Proton-IMPT & Proton-Arc \\
\hline $\mathrm{Cl}$ & $1.30 \pm 0.52$ & $1.29 \pm 0.57$ & $1.28 \pm 0.52$ \\
\hline $\mathrm{HI}$ & $0.74 \pm 0.18$ & $0.47 \pm 0.13^{\star \star \star}$ & $0.48 \pm 0.14^{\star \star \star}$ \\
\hline $\mathrm{V}_{50 \%}\left(\mathrm{~cm}^{3}\right)$ & $1403.82 \pm 610.40$ & $864.86 \pm 449.49^{\star \star \star}$ & $833.51 \pm 381.29^{\star \star \star}$ \\
\hline $\mathrm{V}_{95 \%}\left(\mathrm{~cm}^{3}\right)$ & $402.79 \pm 215.56$ & $389.83 \pm 227.45$ & $395.27 \pm 205.51$ \\
\hline $\mathrm{V}_{100 \%}\left(\mathrm{~cm}^{3}\right)$ & $331.79 \pm 193.25$ & $457.57 \pm 639.63$ & $350.61 \pm 186.75$ \\
\hline
\end{tabular}

Abbreviations: $\mathrm{V}_{\mathrm{x} \%}$ means the volume of all organs (tumor and OARs) that receive $\geq \mathrm{X} \%$ of the prescription dose. Average parameters \pm standard deviation is displayed.

* means $p$ values of $<0.05$ and ${ }^{* *}$ means $p$ values of $<0.001$ compared with Photon-VMAT; There was no statistically significant difference between Proton-IMPT and Proton-Arc. 
We compared esophagus dose in stage N0 to N1 group and N2 to N3 group separately, representing different distances from target to esophagus (Figure 5). In stage N0 to N1 group, $V_{50}$ and $V_{45}$ of esophageal in both proton plans were significantly smaller than Photon-VMAT $(p<0.05)$. However, in stage N2 to N3 group, no significant difference was found in high-dose region of esophageal between proton plans and photon plans ( $p>0.5)$.

Low-dose region analysis (Lung, Spinal cord, Heart)

In the low-dose region, Proton-IMPT and Proton-Arc techniques dramatically reduced the majority OARs dose than Photon-VMAT (<0.05) including ipsilateral lung, spinal cord and heart (Table 4). However, the $\mathrm{V}_{20}$ of contralateral lung in proton plans was not significantly smaller than Photon-VMAT (Photon-VMAT vs. Proton-IMPT ( $p=0.215)$, Photon-VMAT vs. Proton-Arc $(p=0.119)$ ).

Table 4 OARs dose comparison between photon and proton plans 


\begin{tabular}{|c|c|c|c|c|}
\hline OARs & Parameter & Photon-VMAT & Proton-IMPT & Proton-Arc \\
\hline \multirow[t]{3}{*}{ Lungs } & $D_{\text {mean }}(G y E)$ & $11.29 \pm 4.37$ & $5.87 \pm 3.26^{\star \star \star}$ & $5.47 \pm 2.93^{\star \star \star}$ \\
\hline & $V_{5}(\%)$ & $51.65 \pm 20.99$ & $17.57 \pm 10.71^{\star \star \star}$ & $19.31 \pm 11.75^{\star \star \star}$ \\
\hline & $\mathrm{V}_{20}(\%)$ & $16.72 \pm 8.17$ & $10.24 \pm 6.26^{\star \star}$ & $8.36 \pm 5.21^{\star \star \star}$ \\
\hline \multirow[t]{3}{*}{ Ipsilateral lung } & $D_{\text {mean }}(G y E)$ & $18.84 \pm 7.39$ & $12.31 \pm 6.41^{\star \star \star}$ & $11.25 \pm 5.84^{\star \star \star}$ \\
\hline & $\mathrm{V}_{5}(\%)$ & $58.44 \pm 22.02$ & $38.84 \pm 17.90^{\star \star \star}$ & $41.23 \pm 19.06^{* *}$ \\
\hline & $\mathrm{V}_{20}(\%)$ & $35.85 \pm 14.98$ & $23.93 \pm 12.14^{\star \star \star}$ & $20.18 \pm 10.02^{\star \star \star}$ \\
\hline \multirow[t]{3}{*}{ Contralateral lung } & $D_{\text {mean }}(G y E)$ & $6.40 \pm 3.26$ & $0.78 \pm 1.72^{\star \star \star}$ & $0.85 \pm 1.36^{* \star \star}$ \\
\hline & $\mathrm{V}_{5}(\%)$ & $48.66 \pm 22.79$ & $4.31 \pm 8.38^{\star \star \star}$ & $5.61 \pm 9.04^{\star \star \star}$ \\
\hline & $\mathrm{V}_{20}(\%)$ & $4.10 \pm 6.85$ & $1.81 \pm 4.27$ & $1.21 \pm 2.94$ \\
\hline \multirow[t]{3}{*}{ Spinal cord } & $D_{\text {mean }}(G y E)$ & $10.01 \pm 5.44$ & $2.17 \pm 2.95^{\star \star \star}$ & $1.74 \pm 2.04^{\star \star \star}$ \\
\hline & $\mathrm{D}_{\max }(\mathrm{GyE})$ & $33.65 \pm 7.57$ & $22.44 \pm 17.10^{\star \star}$ & $16.66 \pm 14.73^{\star \star \star}$ \\
\hline & $D_{02}(G y E)$ & $28.46 \pm 8.77$ & $12.26 \pm 13.16^{\star \star \star}$ & $10.57 \pm 11.98^{\star \star \star}$ \\
\hline \multirow[t]{4}{*}{ Heart } & $D_{\text {mean }}(G y E)$ & $12.53 \pm 9.32$ & $2.13 \pm 3.01^{\star \star \star}$ & $3.26 \pm 4.68^{\star \star \star}$ \\
\hline & $\mathrm{V}_{30}(\%)$ & $12.31 \pm 12.56$ & $1.72 \pm 2.78^{* \star}$ & $1.49 \pm 2.33^{\star \star}$ \\
\hline & $\mathrm{V}_{40}(\%)$ & $5.45 \pm 6.79$ & $1.00 \pm 1.79^{* \star}$ & $0.85 \pm 1.36^{\star \star}$ \\
\hline & $\mathrm{V}_{45}(\%)$ & $3.58 \pm 4.91$ & $0.76 \pm 1.43$ & $0.66 \pm 1.08$ \\
\hline
\end{tabular}

Abbreviations: $D_{X}$ means the maximize dose received by $X \%$ volume. $V_{X}$ means the percentage of the structure receiving doses greater than $X$ does. Average parameters \pm standard deviation is displayed.

* means $p$ values of $<0.05$, ** means $p$ values of $<0.01$ and $* \star *$ means $p$ values of $<0.001$ compared with Photon-VMAT; There was no statistically significant difference between Proton-IMPT and Proton-Arc.

Clinical benefit for lung and esophagus

While comparing proton to photon plans based on mean NTCP values, the results showed the clinical benefit in terms of the probability of symptomatic radiation pneumonitis and grade $\geq 2$ radiation-induced esophagitis within 6 months of radiation. The estimated NTCP of three groups were shown in figure 6 . As compared with Photon-VMAT, Proton-IMPT decreased the mean probability of symptomatic radiation 
pneumonitis by $61.63 \%$ (from $7.61 \pm 0.91 \%$ to $2.92 \pm 0.34 \% ; p<0.001$ ), and Proton-IMPT could reduce the mean probability of grade $\geq 2$ radiation-induced esophagitis by $50.00 \%$ (from $31.50 \pm 3.75 \%$ to $15.75 \pm 2.78 \% ; p=0.001$ ). (Table 5)

Table 5 NTCP comparison between photon and proton plans

\begin{tabular}{|llll|}
\hline OARs & Photon-VMAT & Proton-IMPT & Proton-Arc \\
\hline Lungs & $7.61 \pm 0.91 \%$ & $2.92 \pm 0.34 \%^{\star \star \star}$ & $2.63 \pm 0.28 \%^{* \star \star}$ \\
\hline Esophagus & $31.50 \pm 3.75 \%$ & $15.75 \pm 2.78 \%^{\star \star \star}$ & $17.69 \pm 2.78 \%^{* \star}$ \\
\hline
\end{tabular}

** means $p$ values of $<0.01$ and $* \star \star$ means $p$ values of $<0.001$ compared with Photon-VMAT; There was no statistically significant difference between Proton-IMPT and Proton-Arc.

\section{Discussion}

Our previous study found that photon P-SABR could improve BED remarkably without increase the OARs dose compared with CFRT, indicating P-SABR might be a safe and effective method for treating large NSCLC. What's more, improving $B_{90}$ could increase the local control rate of NSCLC ${ }^{[13]}$. In this study, we investigate the dosage advantage of proton beam to further increase the target BED.

Proton beam is capable of increasing the target dose regardless of the dose ceiling ${ }^{[23]}$. According to the definition of P-SABR, the center dose of GTVb was as high as possible regardless of homogeneity. Under this condition, proton beam was capable of producing higher dose than photon beam in high-dose region. Proton-IMPT P-SABR can increase $\mathrm{D}_{\text {mean }}, \mathrm{D}_{\text {max }}, \mathrm{B}_{90}$ and $\mathrm{B}_{100}$ by $7.78 \%, 35.56 \%, 15.41 \%$ and $31.81 \%$, respectively compared to Photon-VMAT $(\mathrm{p}<0.05)$. Likewise, in RTOG 0617 trial ${ }^{[24]}$, a phase 3 study on inoperable stage III NSCLC treated by photon CFRT with concurrent chemotherapy, 2-year OS was $57.6 \%$ and $44.6 \%$ for 60GyE and 74GyE arm, respectively. Higher fatal OAR dose, such as heart, might explain why patients given 74 GyE did worse than the 60 GyE dose arm. However, in a phase 2 study of unresectable stage III NSCLC patients treated by proton CFRT (74GyE) with concurrent chemotherapy, 2year OS was as high as $74.5 \%$ while late toxic effects were uncommon ${ }^{[15]}$. These data support increased BED lead to OS benefit in patients treated with proton beams. It's also worth mentioning that BED of 74GyE in conventionally fractionated mode is only $88.8 \mathrm{GyE}$, while the BED of P-SABR center could reach to over $180 \mathrm{GyE}$ in our study, indicating potential clinical beneficial of proton P-SABR.

Similar high-dose of nearby OAR was found between proton with photon plans. Study from Massachusetts General Hospital demonstrated that Proton-IMPT generated larger 95\% isodose volumes when compared with photon SABR account of proton range uncertainty in early-stage NSCLC ${ }^{[16]}$. Nevertheless, according to our research, although Proton-IMPT generated larger $\mathrm{V}_{100 \%}$ by $37.91 \%$ 
$(\mathrm{p}=0.187), \mathrm{V}_{95 \%}$ was albeit smaller by $3.22 \%(\mathrm{p}=0.826)$ and $\mathrm{V}_{50 \%}$ was dramatically smaller by $38.39 \%$ $(p<0.001)$ compared with Photon-VMAT plans. This may due to the photon isodose prescription line was $80 \%$ in their SABR plan but was $100 \%$ in our P-SABR plan. Moreover, research from Niedzielski ${ }^{[25]}$ found there was no significant difference in esophageal toxicity between passively scattered proton therapy and Photon-IMRT therapy. We got similar results that dose of esophageal within high-dose region in photon plans were comparable with proton plans in stage N2 to N3 group ( $p>0.5)$. And N2 to N3 stage NSCLC, to a large extent, means mediastina lymph nodes metastasis, which were more often adjacent to esophagus. Proton beam couldn't decrease the high-dose of nearby esophagus may due to the uncertainty characteristic. We should also note that proton beam doesn't increase the high-dose of nearby OARs.

Due to the feature of Bragg peak, even increasing the target dose, proton therapy is still well known to reduce the "low-dose bath" to normal structures compared with photon therapy. A dosimetric comparison for Proton-IMPT with Photon-IMRT on stage IIIB NSCLC showed that $D_{\text {mean }}, V_{5}, V_{20}$ of both ipsilateral and contralateral lung, $\mathrm{V}_{40}$ of heart and $\mathrm{D}_{\max }$ of spinal cord were significantly lower in Proton-IMPT plan compared with Photon-IMRT plan, even with dose escalation from 63 GyE to $83.5 \mathrm{GyE}$ (median dose of $74 \mathrm{GyE})^{[26]}$, which is similar to the results of our study. Despite this dose escalation advantage of proton P-SABR, $D_{\text {mean }}, V_{5}, V_{20}$ of ipsilateral lung, $D_{\text {mean }}, V_{5}$ of contralateral lung, $D_{\text {mean }}, V_{30}, V_{40}$ of heart and $D_{\text {mean }}, D_{02}, D_{\text {max }}$ of spinal cord were dramatically lower in proton plans compared with Photon-VMAT plans $(P<0.05)$, of which only $V_{20}$ of contralateral lung and $V_{45}$ of heart was not significantly lower in proton plans than Photon-VMAT $(P>0.1)$. In addition, according to NTCP values, proton plans could significantly reduce the probability of symptomatic radiation pneumonitis and grade $\geq 2$ radiationinduced esophagitis within 6 months of radiation over photon plans $(p<0.01)$.

Proton-arc therapy has been assessed in early-stage NSCLC treated by SABR and was able to generate more conformal high-dose volumes than 3 proton passive scattered beams in early-stage NSCLC [17]. So, we included Proton-Arc in our study. Cl was 1.30, 1.29 and 1.28 for Photon-VMAT, Proton-IMPT and Proton-Arc, respectively. Proton-Arc was more conformal slightly ( $p>0.05)$. Nowadays a novel proton arc technique, the spot-scanning proton arc (SPArc) therapy, was able to shorten total treatment delivery time $^{[27]}$ and could provide better target coverage while reduce most of OARs over IMPT in head and neck cancer ${ }^{[28]}$. P-SABR by SPArc technique could be further investigated.

\section{Conclusions}

When applying P-SABR treatment in large NSCLC, proton plans showed dosimetric benefits in increasing target BED, while not improving OAR doses in high-dose region and dramatically minimizing low-dose region OAR doses in comparison to photon plans. Indicating proton P-SABR might further increase local control rate and reduce toxicity at the same time.

\section{Abbreviations}


BED: Biologically effective dose; NSCLC: Non-Small Cell Lung Cancer; P-SABR: Partial Stereotactic Ablative Boost Radiotherapy; CT: computed tomography; Proton-IMPT: intensity modulated proton therapy; Proton-Arc: proton volumetric modulated arc therapy; Photon-VMAT: photon volumetric modulated arc; OARs: Organs-At-Risks; NTCP: Normal tissue complication probability; PTV: Planning Target Volume; SABR: Stereotactic Ablative Radiotherapy; CFRT: conventionally fractionated radiotherapy; GTVb: Gross Tumor boost; GTV: Gross Tumor Volume; ITV: Internal Target Volume; CTV: Clinical Target Volume; AAA: Analytical Anisotropic Algorithm; RBE: Relative biological effectiveness; RTOG: Radiation Therapy Oncology Group; Cl: Conformity index; HI: Homogeneity index; LKB: LymanKutcher-Burman; DVH: dose volume histogram; SPArc: Spot-scanning Proton Arc therapy

\section{Declarations}

Ethics approval and consent to participate

The study was approved by the Peking University First Hospital National Unit of Clinical Trial Ethics Committees. No patient consent was required since this was a retrospective study and the data collection was standard care. All medical data were de-identified.

Consent for publication

Not applicable.

Availability of data and materials

All data generated or analyzed during this study are included in this published article. Additional information is available from the corresponding author on reasonable request.

Competing interests

Not applicable.

Funding

Not applicable.

Authors' contributions

YB contribute to the acquisition, analysis and interpretation of data and draft the manuscript; ZLZ, SWL and $B Z$ contribute to treatment plan analysis, imaging acquisition and support; $S B Q, X Y L$ and $X Q$ contribute to revising the paper and providing clinical inputs; MWM and XYR participate in the study design and draft the manuscript; JYC and MX contribute to the statistical analysis; XSG and XFD contribute to the design of the study, revise the draft and lead the research direction.

Acknowledgements

Page 14/ 24 
Not applicable.

\section{References}

[1] Dubben H H, H D Thames, and H P Beck-Bornholdt. Tumor volume: a basic and specific response predictor in radiotherapy. Radiother Oncol. 1998; 47: 167-74.

[2] Solberg T D, J M Balter, S H Benedict, B A Fraass, B Kavanagh, C Miyamoto, et al. Quality and safety considerations in stereotactic radiosurgery and stereotactic body radiation therapy: Executive summary. Pract Radiat Oncol. 2012; 2: 2-9.

[3] Videtic G M M, J Donington, M Giuliani, J Heinzerling, T Z Karas, C R Kelsey, et al. Stereotactic body radiation therapy for early-stage non-small cell lung cancer: Executive Summary of an ASTRO EvidenceBased Guideline. Pract Radiat Oncol. 2017; 7: 295-301.

[4] Timmerman R, R Paulus, J Galvin, J Michalski, W Straube, J Bradley, et al. Stereotactic body radiation therapy for inoperable early stage lung cancer. Jama. 2010; 303: 1070-6.

[5] Chang J Y, S Senan, M A Paul, R J Mehran, A V Louie, P Balter, et al. Stereotactic ablative radiotherapy versus lobectomy for operable stage I non-small-cell lung cancer: a pooled analysis of two randomised trials. Lancet Oncol. 2015; 16: 630-7.

[6] Woody N M, K L Stephans, G Marwaha, T Djemil, and G M Videtic. Stereotactic Body Radiation Therapy for Non-Small Cell Lung Cancer Tumors Greater Than $5 \mathrm{~cm}$ : Safety and Efficacy. Int J Radiat Oncol Biol Phys. 2015; 92: 325-31.

[7] Allibhai Z, M Taremi, A Bezjak, A Brade, A J Hope, A Sun, et al. The impact of tumor size on outcomes after stereotactic body radiation therapy for medically inoperable early-stage non-small cell lung cancer. Int J Radiat Oncol Biol Phys. 2013; 87: 1064-70.

[8] Cuaron J J, E D Yorke, A Foster, M Hsu, Z Zhang, F Liu, et al. Stereotactic body radiation therapy for primary lung cancers $>3$ centimeters. J Thorac Oncol. 2013; 8: 1396-401.

[9] Zhao J, E D Yorke, L Li, B D Kavanagh, X A Li, S Das, et al. Simple Factors Associated With RadiationInduced Lung Toxicity After Stereotactic Body Radiation Therapy of the Thorax: A Pooled Analysis of 88 Studies. Int J Radiat Oncol Biol Phys. 2016; 95: 1357-66.

[10] Feddock J, S M Arnold, B J Shelton, P Sinha, G Conrad, L Chen, et al. Stereotactic Body Radiation Therapy Can Be Used Safely to Boost Residual Disease in Locally Advanced Non-Small Cell Lung Cancer: A Prospective Study. Int J Radiat Oncol Biol Phys. 2013; 85: 1325-31.

[11] Karam S D, Z D Horne, R L Hong, D McRae, D Duhamel, and N M Nasr. Dose escalation with stereotactic body radiation therapy boost for locally advanced non small cell lung cancer. Radiat Oncol. 2013; 8: 179. 
[12] Peñagarícano J A, E G Moros, V Ratanatharathorn, Y Yan, and P Corry. Evaluation of Spatially Fractionated Radiotherapy (GRID) and Definitive Chemoradiotherapy With Curative Intent for Locally Advanced Squamous Cell Carcinoma of the Head and Neck: Initial Response Rates and Toxicity. Int J Radiat Oncol Biol Phys. 2010; 76: 1369-75.

[13] Bai Y, X S Gao, S B Qin, J Y Chen, M M Su, Q Liu, et al. Partial stereotactic ablative boost radiotherapy in bulky non-small cell lung cancer: a retrospective study. Onco Targets Ther. 2018; 11: 2571-9.

[14] Mohan R and D Grosshans. Proton therapy - Present and future. Adv Drug Deliv Rev. 2017; 109: 2644.

[15] Chang J Y, V Verma, M Li, W Zhang, R Komaki, C Lu, et al. Proton Beam Radiotherapy and Concurrent Chemotherapy for Unresectable Stage III Non-Small Cell Lung Cancer: Final Results of a Phase 2 Study. JAMA Oncol. 2017; 3: e172032.

[16] Seco J, H R Panahandeh, K Westover, J Adams, and H Willers. Treatment of Non-Small Cell Lung Cancer Patients With Proton Beam-Based Stereotactic Body Radiotherapy: Dosimetric Comparison With Photon Plans Highlights Importance of Range Uncertainty. Int J Radiat Oncol Biol Phys. 2012; 83: 354-61.

[17] Seco J, G Gu, T Marcelos, H Kooy, and H Willers. Proton arc reduces range uncertainty effects and improves conformality compared with photon volumetric modulated arc therapy in stereotactic body radiation therapy for non-small cell lung cancer. Int J Radiat Oncol Biol Phys. 2013; 87: 188-94.

[18] Marks L B, E D Yorke, A Jackson, R K Ten Haken, L S Constine, A Eisbruch, et al. Use of normal tissue complication probability models in the clinic. Int J Radiat Oncol Biol Phys. 2010; 76: S10-9.

[19] A N, A M, G LE, G M, L JS, and S HD. Relative biological effectiveness (RBE) values for proton beam therapy. Int J Radiat Oncol Biol Phys. 2002; 53: 407-21.

[20] Mohan R, G S Mageras, B Baldwin, L J Brewster, G J Kutcher, S Leibel, et al. Clinically relevant optimization of 3-D conformal treatments. Med Phys. 1992; 19: 933-44.

[21] Semenenko V A and X A Li. Lyman-Kutcher-Burman NTCP model parameters for radiation pneumonitis and xerostomia based on combined analysis of published clinical data. Phys Med Biol. 2008; 53: 737-55.

[22] Wijsman R, F Dankers, E G Troost, A L Hoffmann, E H van der Heijden, L F de Geus-Oei, et al. Multivariable normal-tissue complication modeling of acute esophageal toxicity in advanced stage nonsmall cell lung cancer patients treated with intensity-modulated (chemo-)radiotherapy. Radiother Oncol. 2015; 117: 49-54.

[23] Choi J I and C B Simone, 2nd. Breaking the dose ceiling: proton therapy for locally advanced nonsmall cell lung cancer. J Thorac Dis. 2018; 10: 130-4. 
[24] Bradley J D, R Paulus, R Komaki, G Masters, G Blumenschein, S Schild, et al. Standard-dose versus high-dose conformal radiotherapy with concurrent and consolidation carboplatin plus paclitaxel with or without cetuximab for patients with stage IIIA or IIIB non-small-cell lung cancer (RTOG 0617): a randomised, two-by-two factorial phase 3 study. Lancet Oncology. 2015; 16: 187-99.

[25] Niedzielski J S, J Yang, R Mohan, U Titt, D Mirkovic, F Stingo, et al. Differences in Normal Tissue Response in the Esophagus Between Proton and Photon Radiation Therapy for Non-Small Cell Lung Cancer Using In Vivo Imaging Biomarkers. Int J Radiat Oncol Biol Phys. 2017; 99: 1013-20.

[26] Zhang X, Y Li, X Pan, L Xiaoqiang, R Mohan, R Komaki, et al. Intensity-modulated proton therapy reduces the dose to normal tissue compared with intensity-modulated radiation therapy or passive scattering proton therapy and enables individualized radical radiotherapy for extensive stage IIIB nonsmall-cell lung cancer: a virtual clinical study. Int J Radiat Oncol Biol Phys. 2010; 77: 357-66.

[27] Ding X, X Li, J M Zhang, P Kabolizadeh, C Stevens, and D Yan. Spot-Scanning Proton Arc (SPArc) Therapy: The First Robust and Delivery-Efficient Spot-Scanning Proton Arc Therapy. Int J Radiat Oncol Biol Phys. 2016; 96: 1107-16.

[28] Liu G, X Li, A Qin, W Zheng, D Yan, S Zhang, et al. Improve the dosimetric outcome in bilateral head and neck cancer (HNC) treatment using spot-scanning proton arc (SPArc) therapy: a feasibility study. Radiation Oncology. 2020; 15.

\section{Figures}


(a) Photon P-SABR Plan

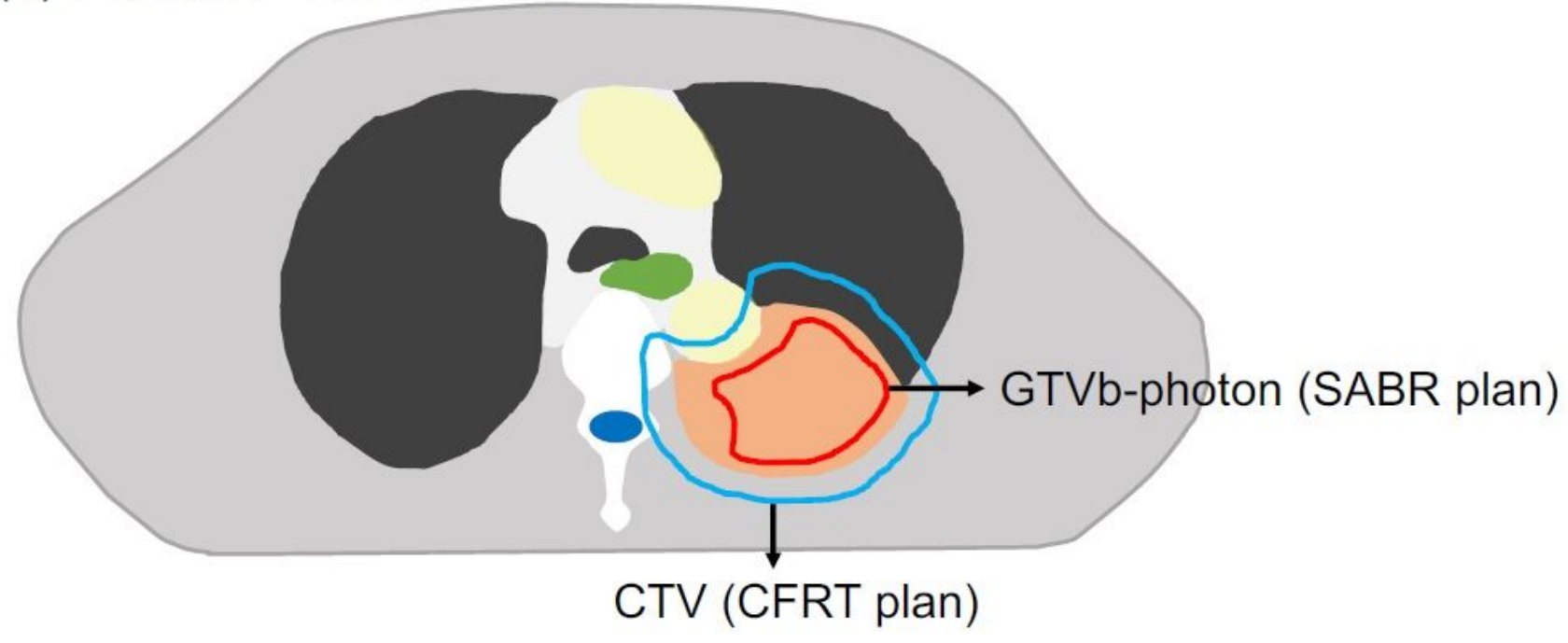

(b) Proton P-SABR Plan

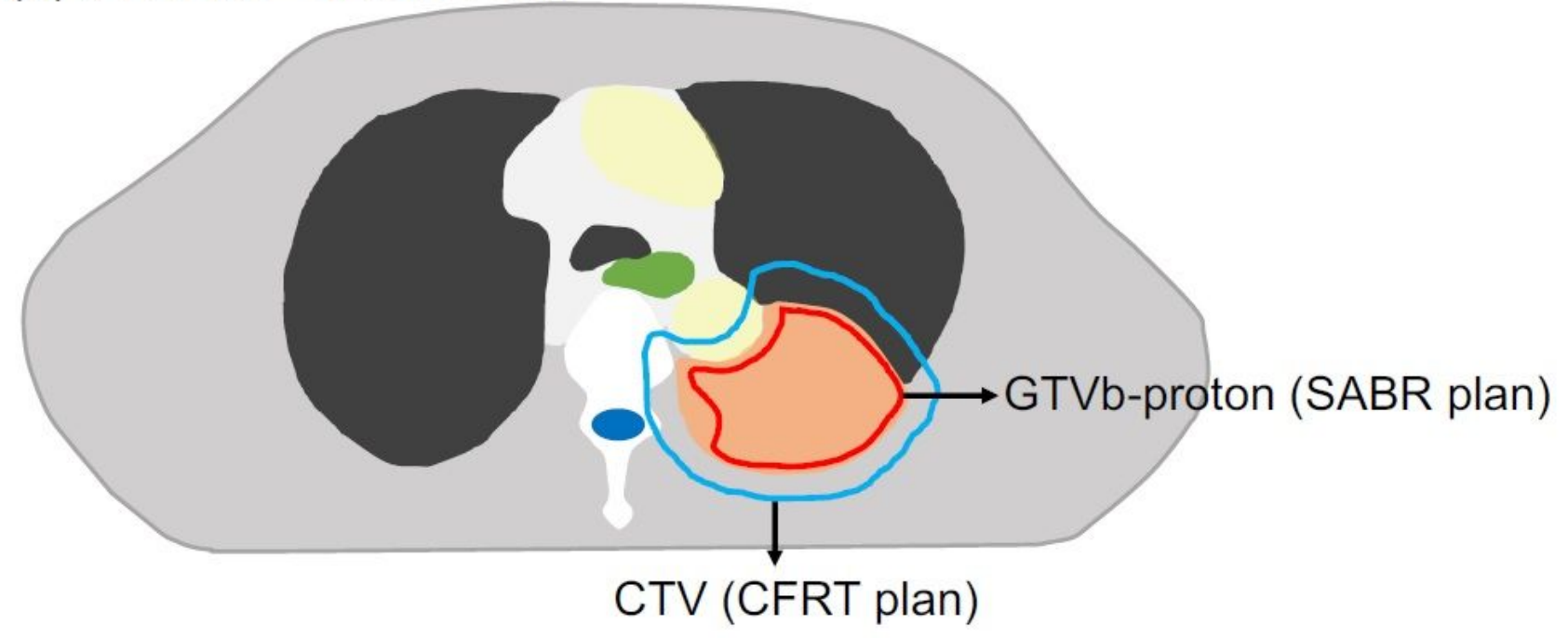

Figure 1

Schematic diagram of the photon (a) and proton (b) P-SABR plan. The P-SABR plan consisted of a partial SABR boost plan to GTVb followed by a CFRT plan to CTV. Individual GTVb-photon and GTVb-proton were the max volume receiving SABR while ensure the fatal OARs dose falloff to $3 \mathrm{GyE} / \mathrm{f}$. For each patient, center dose of GTVb was as high as possible regardless of homogeneity and the total doses of CTV margin were the same. 
(a) Dose

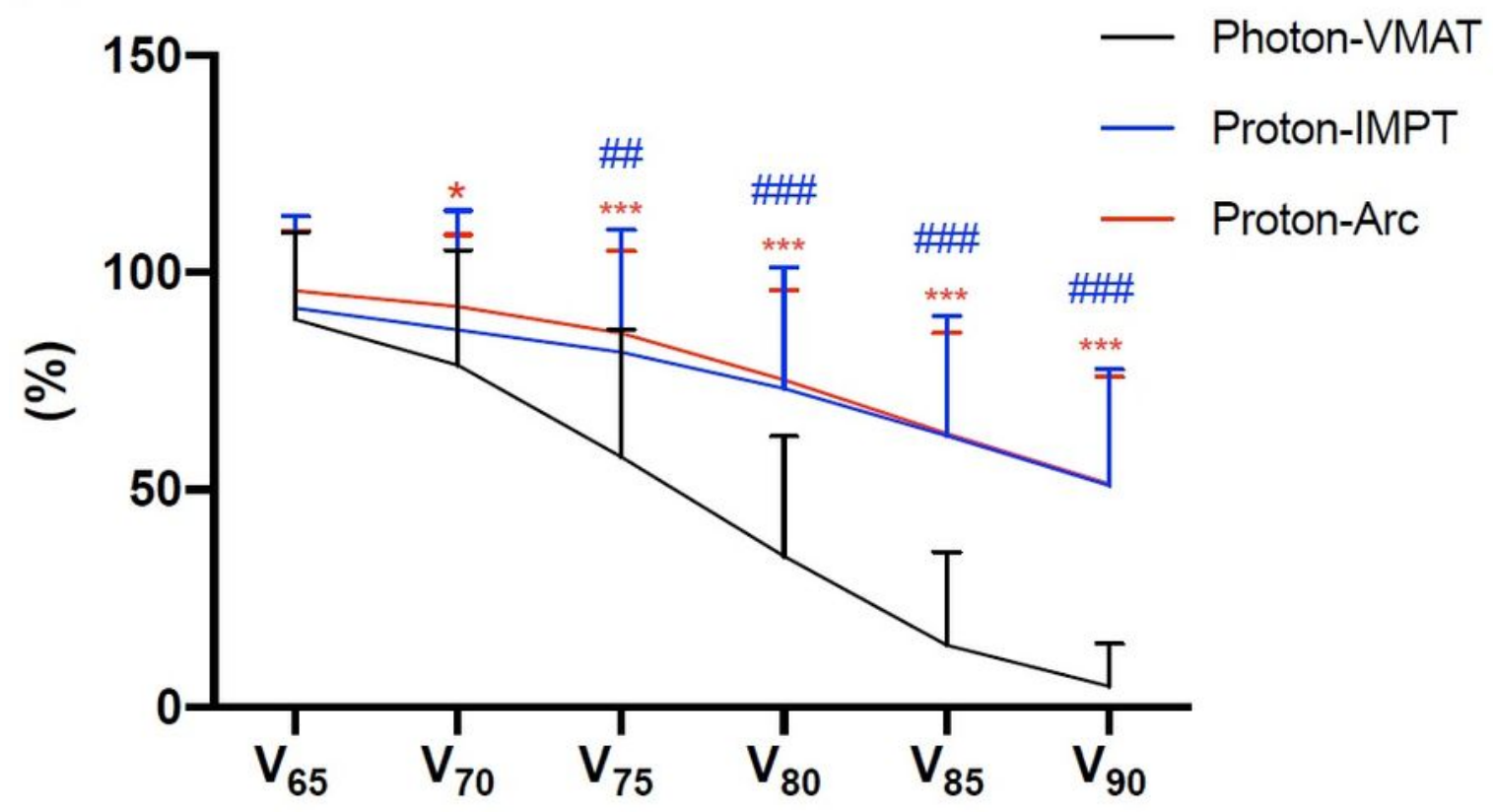

(b)

BED

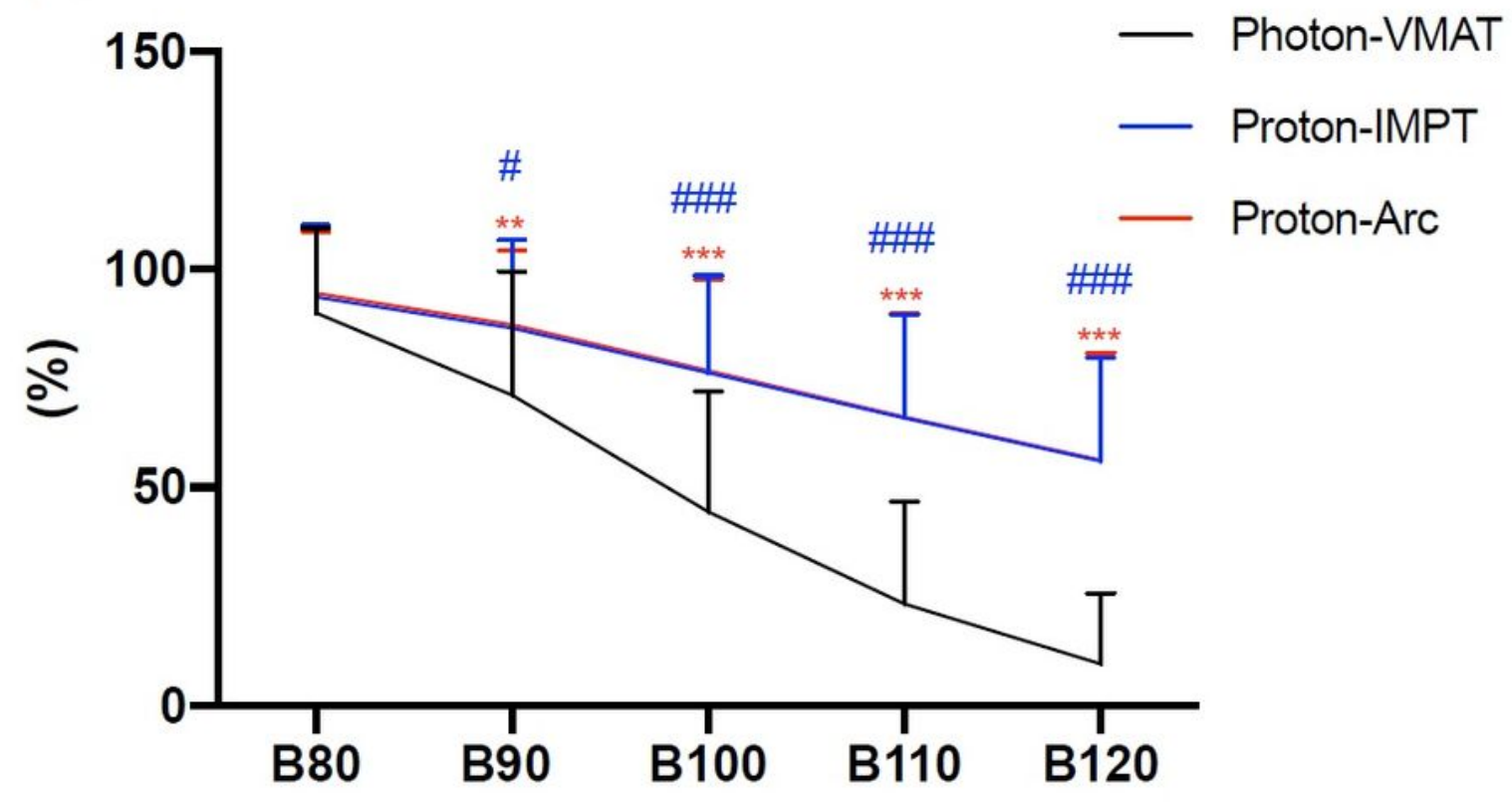

Figure 2

Physical dose (a) and BED (b) of PTV results in Photon-VMAT, Proton-IMPT and Proton-Arc P-SABR plans. $V X$ means the percentage of the structure receiving doses greater than $X$ does. BX means the volume of the structure receiving doses greater than $X$ does; error bars represent maximum values. \# means $p$ values of $<0.05$, \#\# means $p$ values of $<0.01$ and \#\#\# means $p$ values of $<0.001$ compared between Photon-VMAT and Proton-IMPT; * means $p$ values of $<0.05$, ** means $p$ values of $<0.01$ and *** means $p$ 
values of $<0.001$ compared between Photon-VMAT and Proton-Arc; There was no statistically significant difference between Proton-IMPT and Proton-Arc.

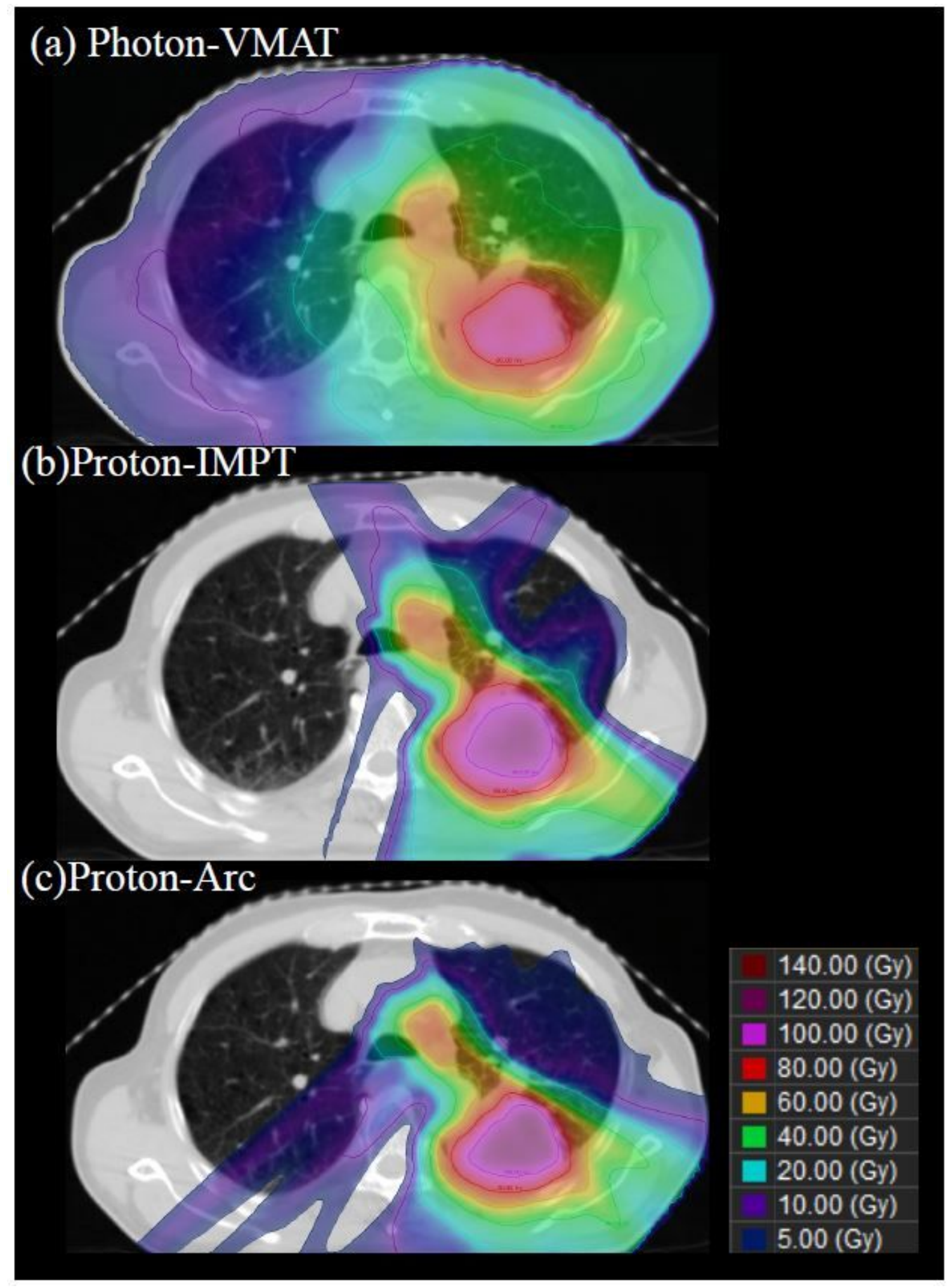

\section{Figure 3}

Photon-VMAT(a), Proton-IMPT(b) and Proton-Arc(c) calculated dose distributions of one patient with large NSCLC treated by P-SABR. 


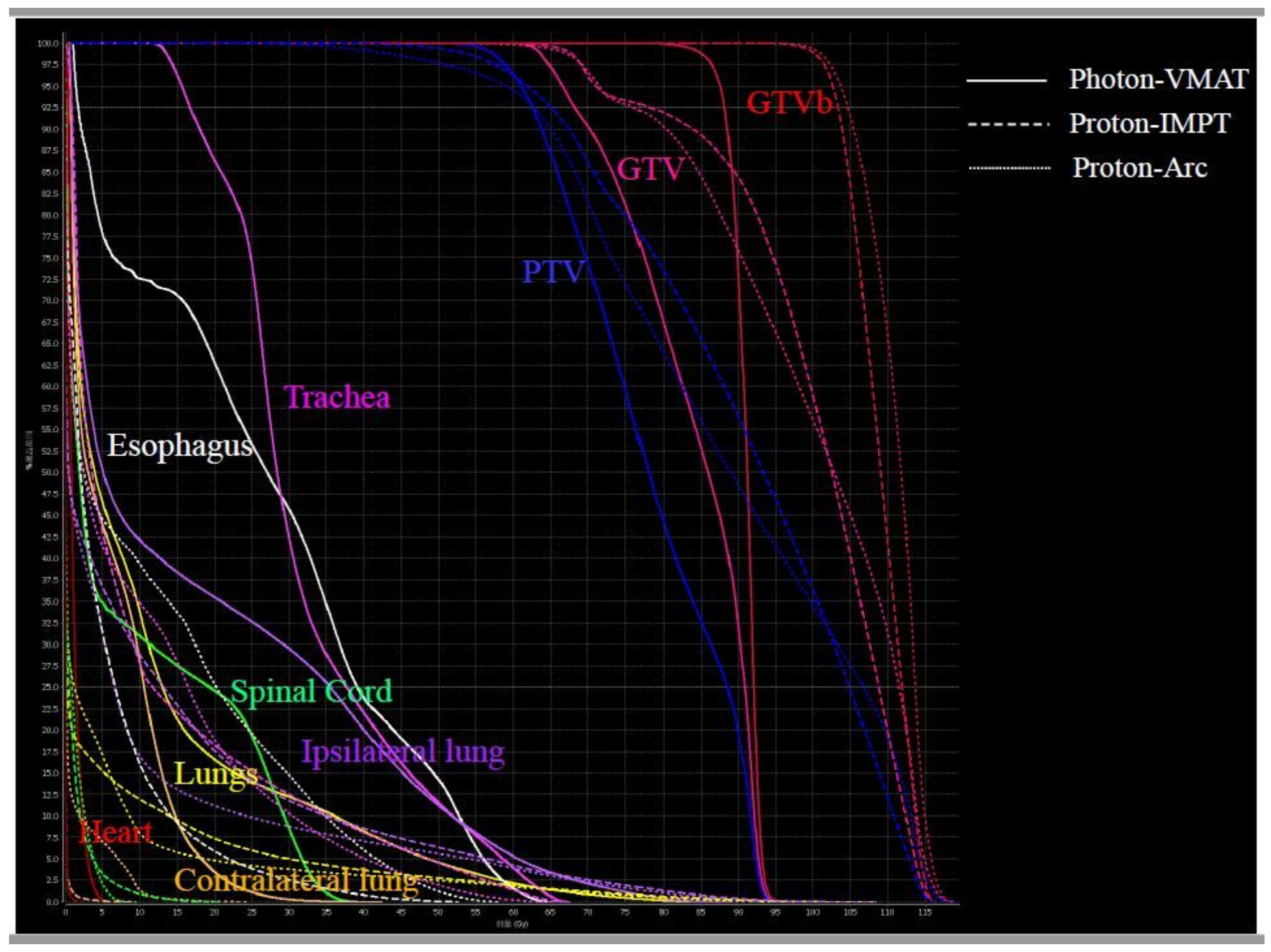

Figure 4

The DVH of an example case for photon P-SABR plans and comparative proton plans. 
(a) Esophagus (N0-1 NSCLC)

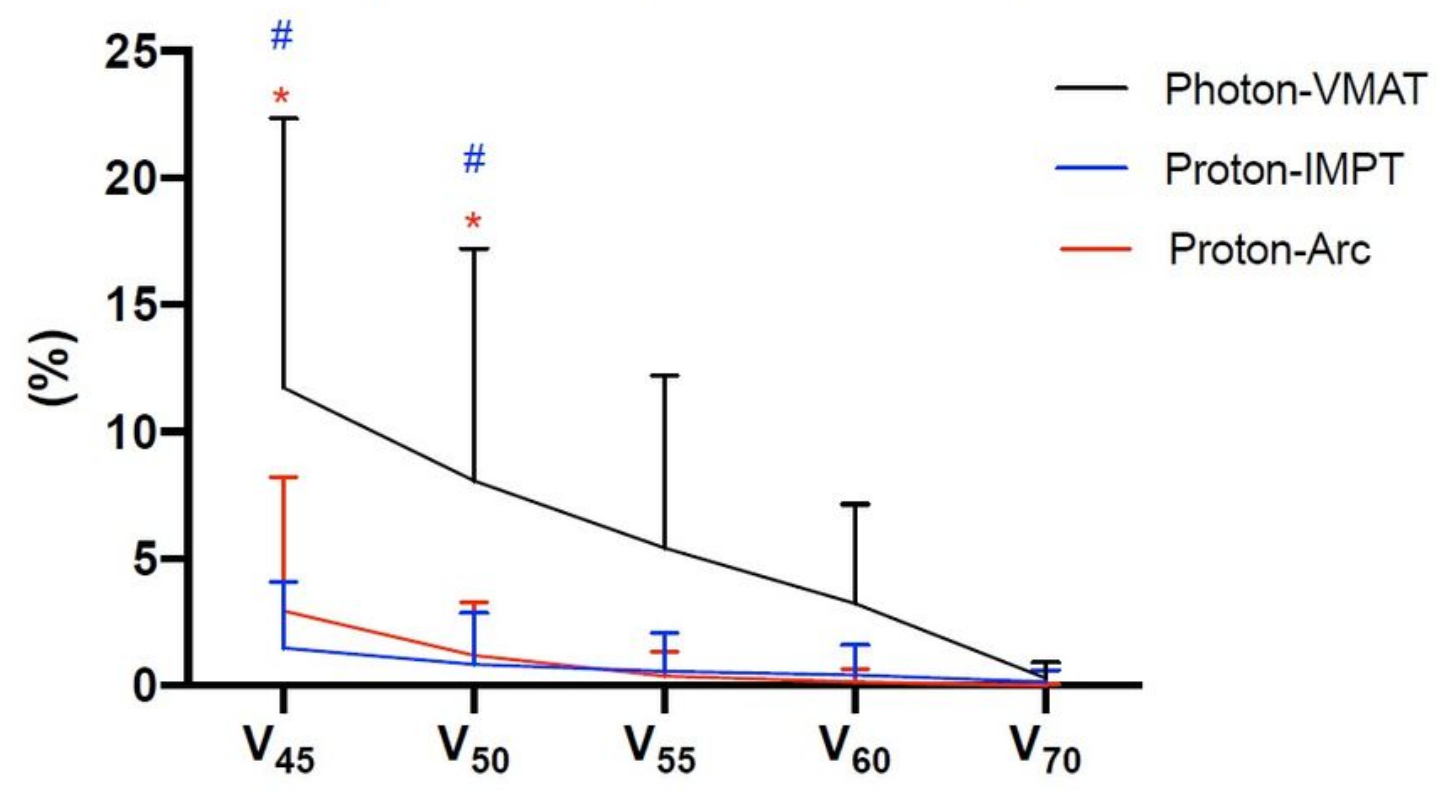

(b) Esophagus (N2-3 NSCLC)

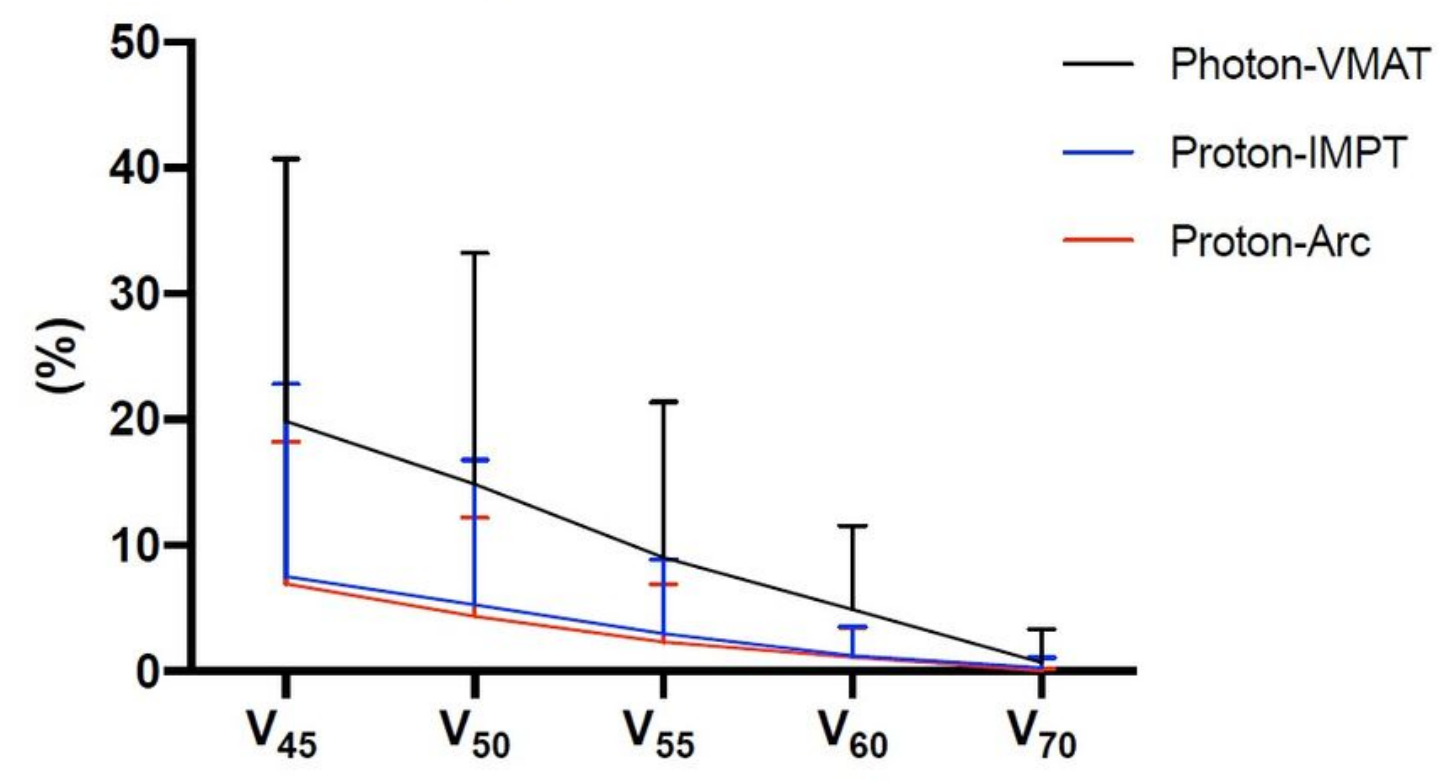

Figure 5

Esophagus dose for Photon-VMAT, Proton-IMPT and Proton-Arc plans were separated into 2 groups, stage N0 to N1 group (a) and N2 to N3 group(b). VX means the percentage of the esophagus receiving doses greater than X GyE does; error bars represent maximum values. \# means $p$ value of $<0.05$ compared between Photon-VMAT and Proton-IMPT; * means $p$ value of $<0.05$ compared between Photon- 
VMAT and Proton-Arc; There was no statistically significant difference between Proton-IMPT and ProtonArc.

(a)

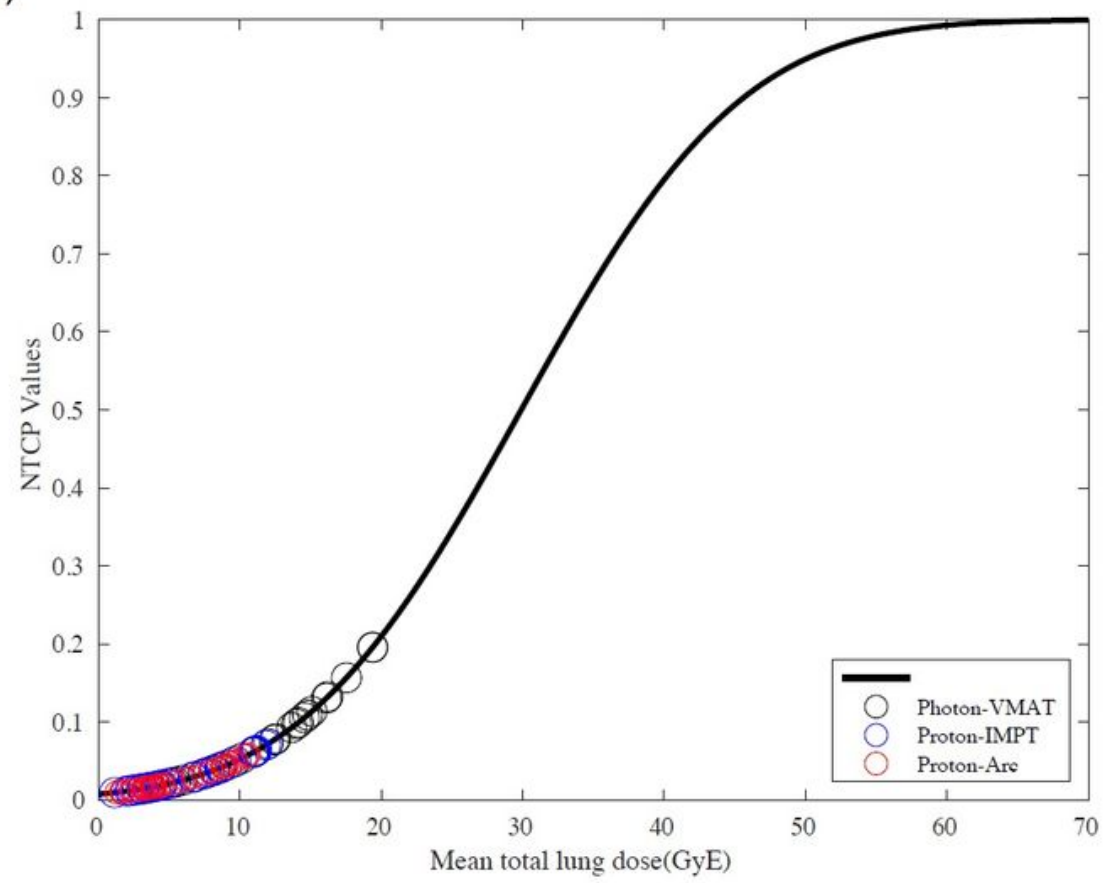

(b)

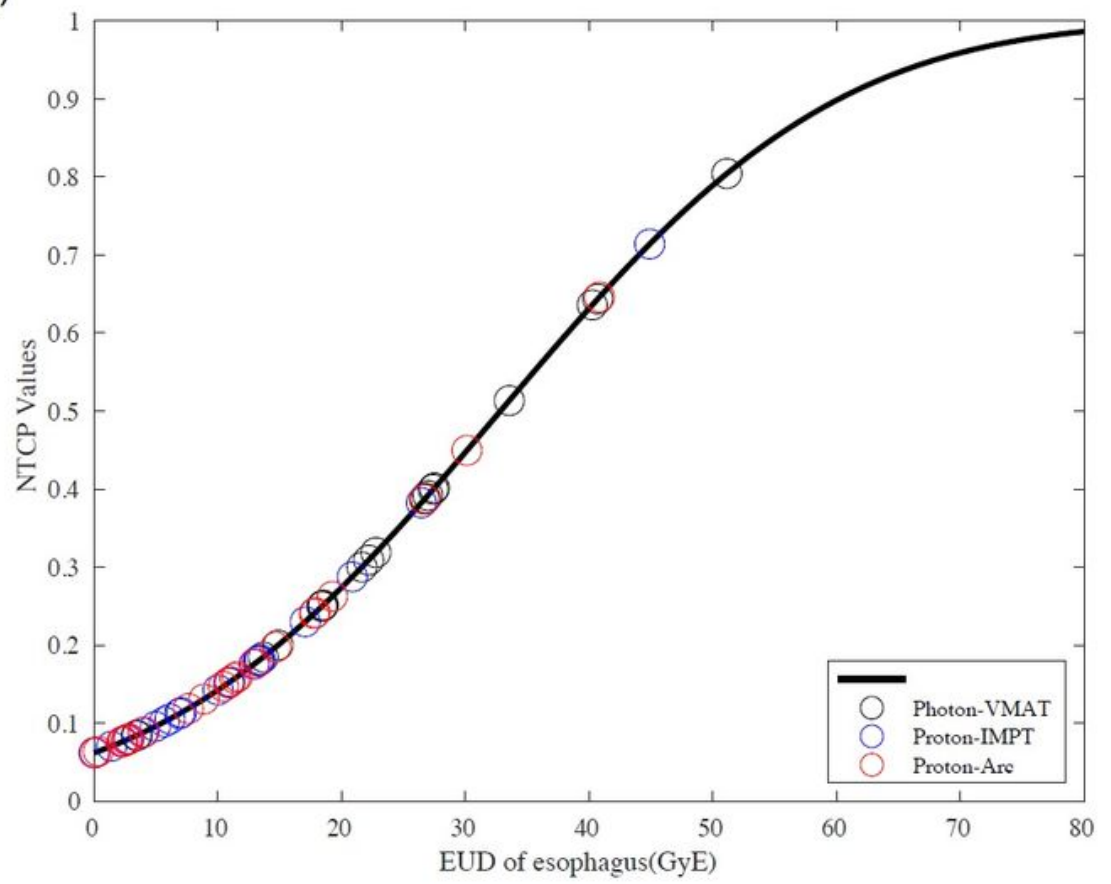

Figure 6

(a) Normal tissue complication probability (NTCP) model for total lung. We used this model to predict the probability of symptomatic radiation pneumonitis within 6 months of radiation. (b) Normal tissue 
complication probability (NTCP) model for esophagus. The NTCP value denotes the probability of grade $\geq 2$ radiation-induced esophagitis within 6 months from the first treatment. 